\title{
Dor pós-operatória em dentes tratados endodonticamente por estudantes de Odontologia, utilizando técnicas manual e reciprocante
}

\author{
Luciéli Andréia Zajkowski*; Samantha Rodrigues Xavier**; Fabio de Almeida Gomes***; Josué \\ Martos****; Melissa Feres Damian****; Patrícia Maria Poli Kopper*****; Fernanda Geraldo \\ Pappen*****
}

\author{
* Estudante, Faculdade de Odontologia, Universidade \\ Federal de Pelotas \\ ** Doutoranda, Programa de Pós-Graduação em Odontologia, \\ Universidade Federal de Pelotas \\ *** Pós-Doutorando, Programa de Pós-Graduação em \\ Odontologia, Universidade Federal de Pelotas, Professor, \\ Faculdade de Odontologia, Universidade de Fortaleza \\ ***** Professor(a), Programa de Pós Graduação em Odontologia, \\ Faculdade de Odontologia, Universidade Federal de Pelotas \\ Professora, Programa de Pós Graduação em Odontologia, \\ Faculdade de Odontologia, Universidade Federal do Rio \\ Grande do Sul
}

Recebido em 03/01/2020. Aprovado em 28/04/2020.

\begin{abstract}
RESUMO
O objetivo do presente estudo foi determinar a frequência e/ou intensidade de dor pós-operatória e os fatores associados a esta ocorrência em tratamentos e retratamentos endodônticos realizados pelas técnicas de instrumentação manual e reciprocante, por estudantes de graduação em Odontologia da Universidade Federal de Pelotas. Para tal, utilizou-se informações dos prontuários de pacientes atendidos por estudantes do último ano de graduação, no período compreendido entre janeiro de 2017 e julho de 2019, totalizando 182 tratamentos. A dor foi registrada por meio da Escala de Avaliação Numérica da Dor (EAND). Considerou-se como variáveis preditivas no desfecho de dor pósoperatória: dente, número de canais, sintomas, coroa dentária, tratamento indicado e índice periapical radiográfico. Como variáveis relativas aos tratamentos, técnica de instrumentação, número de sessões e limite apical de instrumentação. Havia relato de dor no momento do tratamento em $28 \%$ dos prontuários, enquanto $11,5 \%$ não reportaram dor prévia. Em 149 casos $(81,9 \%)$ incluídos neste estudo não foi relatada a ocorrência de dor pós-operatória, enquanto dor leve foi relatada em 17 casos $(9,3 \%)$, moderada em $6(3,3 \%)$ e intensa em $10(5,5 \%)$. Em 67,4\% dos casos dos dentes foram tratados até a marcação 0 do localizador apical, enquanto em $32,6 \%$ o comprimento foi estabelecido a $1 \mathrm{~mm}$ desta marcação. Não houve associação entre o tipo de tratamento realizado e a ocorrência de dor pósoperatória $(\mathrm{p}=0,206)$. Conclui-se que, entre os fatores avaliados, apenas a sintomatologia prévia apresentou associação com a maior frequência de dor pós operatória.
\end{abstract}

Descritores: Ensino Odontológico. Endodontia. Tratamento do Canal Radicular. Odontalgia. 


\section{INTRODUÇÃO}

A endodontia trabalha em regiões inacessíveis ao olho humano, exigindo do profissional um nível apurado de sensibilidade, habilidade e experiência, assim como uma longa curva de aprendizado dos estudantes de Odontologia ${ }^{1}$. Na maioria das escolas de Odontologia brasileiras persiste a utilização da técnica de instrumentação com limas manuais que, devido à sua complexidade e necessidade de sensibilidade tátil elevada, contribui para a ocorrência de uma rejeição natural à Endodontia ${ }^{2}$, e consequente baixa qualidade do tratamento endodôntico realizado por estudantes e profissionais recém-formados ${ }^{3,4}$. A introdução do movimento reciprocante nos cursos de Odontologia abre uma nova perspectiva educacional capaz de levar o profissional a obter preparos com ótimo padrão de modelação, mesmo em casos de maior complexidade ${ }^{5}$, permitindo que mesmo estudantes com pouca experiência possam realizar o tratamento de casos mais complexos ${ }^{6}$.

Além do sucesso clínico e radiográfico, o conforto pós-operatório do paciente é um dos fatores almejados ao se realizar um tratamento ou retratamento endodôntico. Neste contexto, a ocorrência de dor pós-operatória é um problema relativamente frequente para pacientes submetidos à procedimentos endodônticos e vem sendo amplamente estudada ${ }^{7-11}$. Em uma revisão sistemática da literatura ${ }^{7}$ foram avaliados estudos sobre a prevalência de dor antes, durante e após o tratamento endodôntico, tendo sido encontrada prevalência média de $24 \%$ e $11 \%$ após um e sete dias, respectivamente. Tal ocorrência resulta de um processo multifatorial, sendo influenciada por aspectos relacionados ao paciente, ao dente a ser tratado e às habilidades e intervenções do cirurgiãodentista $^{12}$.

Alguns fatores técnicos já demonstraram ter influência sobre a ocorrência de dor após o tratamento de canais radiculares, incluindo instrumentação e/ou obturação insuficiente, extravasamento de solução irrigadora, extrusão apical de detritos e alargamento foraminal durante $o$ preparo do canal radicular ${ }^{13}$. Dentre as principais causas relacionadas à dor pós operatória está a extrusão de debris presentes no canal radicular para os tecidos periapicais ${ }^{14,15}$. A quantidade de debris extruídos e de neuropeptídeos liberados no ligamento periodontal diferem de acordo com a técnica de instrumentação utilizada ${ }^{16}$, o que vem sendo relacionado com os diferentes níveis de intensidade e frequência de dor relatada pelos pacientes.

Desta forma, é objetivo deste estudo determinar a frequência e/ou intensidade de dor pósoperatória e os fatores associados a esta ocorrência em tratamentos e retratamentos endodônticos realizados pelas técnicas de instrumentação manual e reciprocante, estudantes de graduação em Odontologia da Universidade Federal de Pelotas (UFPel).

\section{METODOLOGIA \\ Desenho experimental}

Este foi um estudo retrospectivo, utilizando informações disponíveis em prontuários de pacientes atendidos por estudantes do último ano de graduação na Faculdade de Odontologia da UFPel, no período compreendido entre janeiro de 2017 e julho de 2019, aprovado pelo Comitê de Ética em Pesquisa institucional (CAAE: 06198819.6.2001. 5317).

Foram incluídos dados da anamnese e dos exames clínico e radiográficos dos pacientes atendidos, níveis de dor pré e pós-operatória. Os dados obtidos nos prontuários relativos ao diagnóstico, ao tratamento e às escalas de dor, foram tabulados e armazenados em um banco de dados.

\section{Variáveis incluídas no estudo}

As variáveis preditivas no desfecho dor pósoperatória foram: dente (grupo dentário: anteriores, pré-molares ou molares), número de canais, 
sintomas (ausentes, ausentes com antecedentes ou presentes), coroa dentária (hígida, restaurada, cariada, dente previamente abordado), tratamento indicado (biopulpectomia, necropulpectomia ou retratamento) e índice periapical radiográfico (1: estrutura óssea periapical normal; 2: pequenas alterações na estrutura óssea, sem desmineralização; 3: alterações na estrutura óssea, com perda mineral difusa; 4: periodontite apical, com área radiolúcida bem definida; 5: periodontite apical severa, com características de exacerbação radiográfica). Como variáveis relativas ao tratamento, foram consideradas: técnica de instrumentação (manual ou reciprocante), número de sessões (única, 2 sessões, 3 ou mais sessões) e limite apical de instrumentação (1 mm aquém da marcação 0 do localizador apical com ou sem patência; ou na marcação 0 do localizador apical).

\section{Tratamento endodôntico}

Todos os procedimentos foram realizados sob condições padronizadas, e sob supervisão de um professor de Endodontia.

Os canais radiculares foram preparados utilizando uma das seguintes técnicas: manual (técnica coroa-ápice com instrumentos manuais de aço inoxidável) ou reciprocante (sistemas WaveOne Gold ou Reciproc, segundo instruções do fabricante). A irrigação durante o preparo biomecânico foi realizada com hipoclorito de sódio $(\mathrm{NaOCl})$ a 2,5\% e ácido etilenodiamino tetraacético (EDTA) a $17 \%$ por 3 a 5 minutos ao final do preparo. Nos casos em que mais de uma sessão foi necessária, foi utilizada medicação intracanal à base de hidróxido de cálcio. A obturação foi realizada pela técnica do cone único dos sistemas WaveOne Gold ou Reciproc, ou técnica de condensação lateral da guta-percha, no caso da instrumentação manual.

\section{Avaliação da dor}

Para avaliação da presença e intensidade da dor pré e pós-operatória foi aplicada a Escala de Avaliação Numérica da Dor (EAND), validada para língua portuguesa ${ }^{17}$. A EAND consiste em uma escala de 11 pontos apresentados, frequentemente, em caixotes fechados em ordem crescente de números inteiros da esquerda para a direita de 0 à 10 , na qual os pontos extremos significam "sem dor" para 0 e "pior dor que se pode imaginar" para 10. Os participantes serão solicitados a assinalar com um " $\mathrm{x}$ " o único número que melhor representa a intensidade da dor. A escala foi aplicada em momentos distintos: na primeira consulta durante o exame clínico completo e anamnese; após o preparo biomecânico e colocação da medicação intracanal (nos casos onde não foi concluído o tratamento); e após a obturação dos canais radiculares.

$\mathrm{Na}$ primeira consulta após exame clínico completo, os pacientes foram questionados quanto à intensidade da experiência de dor de origem dentária nas últimas 48 horas (dor pré-operatória), sendo orientados e solicitados a registrar na escala o número correspondente à intensidade de dor. Após o término da consulta, os pacientes levaram para casa uma escala impressa, sendo instruídos a preenchê-las quanto a presença e intensidade da sintomatologia nas primeiras 24 e 48 horas após cada sessão clínica. Quando não houve consulta presencial os pacientes foram ainda contatados via ligação telefônica em horários pré-estabelecidos. Para fins estatísticos, os dados numéricos referentes à dor pós-operatória foram categorizados em $0=$ ausente; 1 a $4=$ dor leve; 5 a $7=$ dor moderada; 8 a $10=$ dor intensa).

\section{Análise dos dados}

A análise estatística foi realizada utilizando o software SPSS v. 22.0 (SPSS Inc, Chicago, IL). Foram realizadas análises descritivas dos dados referentes aos dentes incluídos no estudo, e índices de dor pré e pós-operatória, além de testes de associação entre as variáveis e o desfecho endodôntico. 


\section{RESULTADOS}

A frequência e a intensidade de dor pósoperatória e sua associação com as principais variáveis avaliadas está descrita na tabela 1. Durante o período de avaliação, foram finalizados 182 tratamentos endodônticos pelos estudantes do último ano do curso. Em 149 $(81,9 \%)$ destes 182 casos incluídos no estudo não foi relatada a ocorrência de dor em nenhum dos períodos de aferição pós-operatória, enquanto dor leve foi relatada em 17 casos $(9,3 \%)$, moderada em $6(3,3 \%)$, e intensa em $10(5,5 \%)$. Do total de casos, $57(31,1 \%)$ eram de dentes com vitalidade pulpar, $88(48,4 \%)$ de dentes sem vitalidade e 37 (20,3\%) eram casos de retratamento.

Tabela 1. Frequência dor pós-operatória e sua associação com as principais variáveis avaliadas

\begin{tabular}{|c|c|c|c|c|c|c|}
\hline & \multicolumn{6}{|c|}{ Dor n (\%) } \\
\hline & Total & Ausente & Leve & Moderada & Severa & Valor de $P$ \\
\hline \multicolumn{7}{|l|}{ Instrumentação } \\
\hline Manual & $46(100)$ & $36(78,3)$ & $6(13)$ & $2(4,3)$ & $2(4,3)$ & 0,733 \\
\hline Reciprocante & $132(100)$ & $110(83,3)$ & $11(8,3)$ & $4(3)$ & $7(5,3)$ & \\
\hline \multicolumn{7}{|l|}{ Dente } \\
\hline Anterior & $72(100)$ & $64(88,9)$ & $3(4,2)$ & $2(2,8)$ & $3(4,2)$ & 0,135 \\
\hline Pré-molar & $40(100)$ & $32(80)$ & $4(10)$ & $2(5,0)$ & $2(5,0)$ & \\
\hline Molar & $69(100)$ & $52(75,4)$ & $10(14,5)$ & $2(2,9)$ & $5(7,2)$ & \\
\hline \multicolumn{7}{|l|}{ Tratamento } \\
\hline Biopulpectomia & $57(100)$ & $44(77,2)$ & $5(8,8)$ & $3(5,3)$ & $5(8,8)$ & 0,206 \\
\hline Necropulpectomia & $88(100)$ & $75(85,2)$ & $7(8,0)$ & $2(2,3)$ & $4(4,5)$ & \\
\hline Retratamento & $37(100)$ & $30(81,1)$ & $5(13,5)$ & $1(2,7)$ & $1(2,7)$ & \\
\hline \multicolumn{7}{|l|}{ Sintomas iniciais } \\
\hline Ausentes & $107(100)$ & $97(90,7)$ & $8(7,5)$ & $1(0,9)$ & $1(0,9)$ & 0,000 \\
\hline Presentes & $54(100)$ & $36(66,7)$ & $6(11,1)$ & $3(5,6)$ & $9(16,7)$ & \\
\hline Dor prévia & $21(100)$ & $16(76,2)$ & $3(14,3)$ & $2(9,5)$ & $0(0,0)$ & \\
\hline \multicolumn{7}{|l|}{ PAI inicial } \\
\hline 1 & $91(100)$ & $75(82,4$ & $8(8,8)$ & $4(4,4)$ & $4(4,4)$ & 0,484 \\
\hline 2 & $29(100)$ & $24(82,8)$ & $3(10,3)$ & $0(0,0)$ & $2(6,9)$ & \\
\hline 3 & $27(100)$ & $24(88,9)$ & $1(3,7)$ & $1(3,7)$ & $1(3,7)$ & \\
\hline 4 & $32(100)$ & $24(75,0)$ & $5(15,6)$ & $0(0,0)$ & $3(9,4)$ & \\
\hline 5 & $3(100)$ & $2(66,7)$ & $0(0,0)$ & $1(33,3)$ & $0(0,0)$ & \\
\hline \multicolumn{7}{|l|}{ Número de sessões } \\
\hline Sessão única & $110(100)$ & $95(86,4)$ & $6(5,5)$ & $2(1,8)$ & $7(6,4)$ & 0,541 \\
\hline 2 sessões & $58(100)$ & $43(74,1)$ & $9(15,5)$ & $3(5,2)$ & $3(5,2)$ & \\
\hline 3 ou mais sessões & $14(100)$ & $11(78,6)$ & $2(14,3)$ & $1(7,1)$ & $0(0,0)$ & \\
\hline
\end{tabular}

Um total de 54 pacientes $(28 \%)$ relatou dor no momento do tratamento, enquanto $21(11,5 \%)$ reportaram dor prévia. A presença de dor nas $48 \mathrm{~h}$ antes da consulta inicial, seja no momento do tratamento ou prévia, apresentou relação estatisticamente significativa $(\mathrm{p}<0,000)$ com o relato de dor pós-operatória de qualquer intensidade.
Em $132(72,5 \%)$ dos casos os estudantes optaram por utilizar instrumentos reciprocantes para o preparo dos canais radiculares e em $46(25,3 \%)$ foram utilizados instrumentos manuais convencionais. A técnica de instrumentação utilizada não interferiu na ocorrência de dor pósoperatória, sendo que índices semelhantes de dor 
leve, moderada ou severa foram relatados para dentes instrumentados pelas técnicas manual e reciprocante $(\mathrm{p}=0,733)$.

Com a introdução das técnicas mecanizadas no ensino de graduação, houve também o aumento da frequência do uso do localizador foraminal, o que permitiu que $122(67,4 \%)$ dos casos fossem tratados até a mensuração 0 do localizador apical, limpando o canal radicular em todo seu comprimento. O limite de instrumentação utilizado, se na mensuração 0 do localizador foraminal ou se $1 \mathrm{~mm}$ aquém dessa medida, não influenciou na ocorrência de dor pósoperatória $(\mathrm{p}=0,729)$.

Não houve associação entre a ocorrência de dor e o grupo dentário tratado, apesar de ser possível observar um percentual maior de dor leve ou moderada em dentes molares e pré-molares $(p$ $=0,135)$. Também o número de canais não esteve associado à maior ocorrência de dor pós-operatória $(\mathrm{p}=0,235)$.

A frequência e intensidade de dor pósoperatória variou de acordo com o tratamento realizado: não houve dor em 44 (77,2\%) dos casos de biopulpectomias, $75(85,2 \%)$ dos casos de necropulpectomias e em $30 \quad(81,1 \%)$ dos retratamentos, porém, não houve associação estatisticamente significativa entre o tratamento realizado e a ocorrência de dor pós operatória $(p=0,206)$. Não houve associação entre as condições da coroa dentária no momento do tratamento (restaurada, previamente abordado, cariado, hígido), o PAI inicial ou o número de sessões necessárias para finalização do tratamento com a ocorrência de dor pós-operatória ( $\mathrm{p}>0,05)$.

\section{DISCUSSÃO}

O objetivo deste estudo foi determinar a frequência de dor pós-operatória e os fatores associados a esta ocorrência em pacientes que receberam tratamento endodôntico realizados por alunos de graduação. Devido à natureza subjetiva e multifatorial da dor, muitas dificuldades podem surgir na medição do nível de dor pós-operatório e no controle dos vários fatores de confusão envolvidos.

$\mathrm{Na}$ avaliação da dor, tentou-se objetivar um fenômeno que é essencialmente subjetivo e sujeito à alta variabilidade individual, ficando clara a complexidade de mensurar resultados referentes à avaliação de dor. O presente estudo utilizou a EAND para mensuração da dor pré e pós-operatória, sendo esta escala uma das ferramentas mais utilizadas para tal avaliação em procedimentos clínicos $^{18-20}$, anteriormente validada para o idioma português ${ }^{17}$.

A dor após o tratamento do canal radicular pode ser relacionada a causas variáveis. No entanto, um dos fatores preditivos para sua ocorrência parece ser a inflamação causada pelos debris extruídos para os tecidos periapicais ${ }^{7,21-23}$. Apesar da literatura apontar que a técnica manual parece extruir maiores quantidades de debris que a reciprocante, no presente estudo não houve diferença significativa da dor pós-operatória entre as diferentes técnicas de instrumentação (manual e reciprocante). Contrariando este resultado, vários estudos ${ }^{24-28}$ têm demonstrado maior ocorrência de dor pós-operatória nos casos em que e utiliza a instrumentação manual, em comparação à instrumentação mecanizada. Tais resultados podem ser atribuídos ao fato de que no presente estudo a técnica manual utilizada foi a coroa-ápice, sendo que a maioria dos estudos que demonstram maior ocorrência de dor pós-operatória nos casos tratados pelas técnicas manuais utilizaram a técnica ápice-coroa ${ }^{26-30}$. Na técnica ápice-coroa existe a tendência do instrumento utilizado agir como um êmbolo, direcionando debris e forçando sua extrusão pelo forame apical ${ }^{31}$, provavelmente exacerbando o quadro inflamatório periapical e, consequentemente, a dor.

A literatura que busca a associação da condição pulpar com a ocorrência de dor pósoperatória ainda fornece dados inconsistentes. Vários estudos relatam que a condição pulpar 
contribui para a dor pós-operatória ${ }^{32,33}$ enquanto outros não ${ }^{8,23,34,35}$. Os resultados do presente estudo, no entanto, demonstraram associação significativa entre a sintomatologia inicial e a ocorrência de dor pós-operatória, o que está de acordo com os resultados previamente apresentados na literatura ${ }^{23,36,37}$. Pacientes que já apresentam dor previamente ao tratamento endodôntico já apresentam algum grau de inflamação dos tecidos pulpares e/ou periapicais, e após o preparo e obturação dos canais radiculares, mesmo que sejam utilizadas substâncias anti-inflamatórias, a inflamação e consequentemente a dor é diminuída de forma gradual, justificando os resultados descritos.

Também foi verificado que o grupo dentário não influenciou na dor pós-operatória, embora já tenha sido reportado que os dentes molares seriam os mais propensos a apresentar dor pós-operatória ${ }^{38}$. Tal associação, provavelmente se deve ao fato que os molares apresentam anatomia mais complexa, com maior número de canais, e curvaturas mais acentuadas, assim como variações anatômicas que podem contribuir para manutenção de paredes sem limpeza e, consequentemente, persistente infecção e inflamação tecidual, mesmo após o preparo biomecânico $^{37-40}$.

O número de sessões em que foram realizadas o tratamento endodôntico não influenciou na dor pós-operatória nesse trabalho, o que está de acordo com estudos anteriores, os quais apontam que o resultado do tratamento endodôntico em uma única sessão ou em várias sessões, em relação à ocorrência de dor, foi semelhante, sem flare-ups e complicações ${ }^{41-43}$. No entanto, a literatura existente também é controversa em relação a estes resultados. Alguns autores apontam um menor índice de dor pós-operatória em tratamentos endodônticos realizados em sessão única ${ }^{44-45}$, enquanto outros apontam um menor índice de dor pós-operatória em múltiplas sessões ${ }^{46-47}$. A menor incidência de dor pós-operatória no tratamento do canal radicular em sessão única poderia ser atribuída à obturação imediata, evitando assim a passagem de medicamentos, instrumentação repetida e irrigação. Além disso, um tratamento endodôntico em única sessão, também poderia impedir a ocorrência de dor resultante da reinfecção dos canais como consequência do ingresso bacteriano frente a uma restauração temporária insatisfatória ${ }^{48}$.

As taxas de dor pós-operatória no presente estudo foram relativamente baixas. Isso pode estar associado aos operadores que realizaram os tratamentos endodônticos, os quais eram estudantes de graduação. Estudos anteriores ${ }^{12,49}$ apontam menor prevalência de dor pós-operatória em tratamentos endodônticos realizados por estudantes de graduação quando comparados a pósgraduandos. Tais achados não podem ser justificados de forma direta, e deve-se levar em consideração as limitações do presente estudo, o qual incluiu múltiplos operadores, o que pode significar uma grande variação em relação aos outros estudos.

\section{CONCLUSÃO}

Os resultados do presente estudo não demonstraram associação entre a dor pósoperatória em dentes tratados endodonticamente por estudantes de graduação e a técnica de instrumentação utilizada, manual ou reciprocante. Dos fatores avaliados, apenas a sintomatologia prévia apresentou associação com a maior frequência e intensidade de dor pós-operatória.

\section{ABSTRACT \\ Postoperative pain in teeth endodontically treated by dental students, using manual and reciprocating techniques \\ This study determined the frequency and/or intensity of postoperative pain and factors associated with such occurrence in endodontic treatments and retreatments performed by manual and reciprocating techniques, by}


undergraduate dental students at the Federal University of Pelotas. For that purpose, data were collected from the records of patients treated by last-term undergraduate students, in the period between January 2017 and July 2019, adding up to 182 treatments. Pain was recorded by the Numerical Pain Rating Scale (NPRS). The following predictive variables were considered in the outcome of postoperative pain: tooth, number of canals, symptoms, dental crown, indicated treatment and radiographic periapical index. The variables related to the treatments included the instrumentation techniques, number of sessions and apical limit of instrumentation. There was report of pain during treatment in $28 \%$ of records, while $11.5 \%$ did not report previous pain. In 149 cases $(81.9 \%)$ included in this study there were no reports of postoperative pain, while mild pain was reported in 17 cases $(9.3 \%)$, moderate in 6 $(3.3 \%)$ and intensive in 10 (5.5\%). In $67.4 \%$ of cases, the teeth were treated up to mark " 0 " of the apex locator, while in $32.6 \%$ the length was established at $1 \mathrm{~mm}$ from this mark. There was no association between the type of treatment performed and the occurrence of postoperative pain $(\mathrm{p}=0.206)$. It was concluded that, among the evaluated factors, only the previous symptomatology was associated with higher frequency of postoperative pain.

Descriptors: Education, Dental. Endodontics. Root Canal Treatment. Toothache.

\section{REFERÊNCIAS}

1. Souza E, Rodrigues E, De-Deus G, Zuolo M, Silva E, Versiani M. Movimento reciprocante e a curva de aprendizagem do preparo mecânico. O Movimento Reciprocante na Endodontia. São Paulo: Quintessence Editora; 2017. p. 214-31.

2. Seijo MO, Ferreira EF, Ribeiro Sobrinho AP, Paiva SM, Martins RC. Learning experience in endodontics: Brazilian students' perceptions. J Dent Educ. 2013;77(5):648-55.

3. Abu-tahun I, Al-Rabab'ah MA, Hammad M, Khraisat A. Technical quality of root canal treatment of posterior teeth after rotary or hand preparation by fifth year undergraduate students, The University of Jordan. Aust Endod J. 2014;40(3):123-30.

4. Er O, Sagsen B, Maden M, Cinar S, Kahraman Y. Radiographic technical quality of root fillings performed by dental students in Turkey. Int Endod J. 2006;39(11):867-72.

5. Rodrigues E, De-Deus G, Souza E, Silva EJ. Safe Mechanical Preparation with reciprocation movement without glide path creation: result from a pool of 673 root canals. Braz Dent J. 2016;27(1):22-7.

6. Muñoz E, Forner L, Llena C. Influence of operator's experience on root canal shaping ability with a rotary nickel-titanium single-file reciprocating motion system. J Endod. 2014;40(4):547-50.

7. Pak JG, White SN. Pain prevalence and severity before, during, and after root canal treatment: A systematic review. J Endod. 2011;37(4):429-38.

8. Pasqualini D, Mollo L, Scotti N, Cantatore G, Castelucci A, Migliaretti G, Berutti E. Postoperative pain after manual and mechanical glide path: a randomized clinical trial. J Endod. 2012;38(1):32-6.

9. Silva EJ, Menaged K, Ajuz N, Monteiro MR, Coutinho-Filho T de S. Postoperative pain after foraminal enlargement in anterior teeth with necrosis and apical periodontitis: a prospective and randomized clinical trial. J Endod. 2013;39(2):173-6.

10. Arias A, Macorra JC, Azabal M, Hidalgo JJ, Peters OA. Prospective case controlled clinical study of post-endodontic pain after rotary root canal preparation performed by a single operator. J Dent. 2015;43(3):389-95.

11. Nekoofar MH, Sheykhrezae MS, Meraji N, Jamee A, Shirvani A, Jamee J, Dummer P. Comparison of the effect of root canal preparation by using WaveOne and ProTaper on postoperative pain: a randomized clinical 
trial. J Endod. 2015;41(5):575-8.

12. Walton R, Fouad A. Endodontic interappointment flare-ups: a prospective study of incidence and related factors. $\mathbf{J}$ Endod. 1992;18(4):172-7.

13. Alves VO. Endodontic flare-ups: a prospective study. Oral Surg Oral Med Oral Pathol Oral Radiol Endod. 2010;110(5):e6872.

14. Seltzer S, Naidorf IJ. Flare-ups in endodontics: I. Etiological factors. J Endod 1985;11(11):472-8.

15. Siqueira Jr JF. Microbial causes of endodontic flare-ups. Int Endod J. 2003;36(7):453-63.

16. Caviedes-Bucheli J, Castellanos F, Vasquez $\mathrm{N}$, Ulate E, Munoz HR. The influence of two reciprocating single-file and two rotary-file systems on the apical extrusion of debris and its biological relationship with symptomatic apical periodontitis. A systematic review and meta-analysis. Int Endod J. 2016;49(3):25570.

17. Ferreira-Valente MA, Pais-Ribeiro JL, Jensen MP. Validity of four pain intensity rating scales. Pain. 2011;152(10):2399-404.

18. Williamson A, Hoggart B. Pain: a review of three commonly used pain rating scales. J Clin Nurs. 2005;14(7):798-804.

19. Hjermstad MJ, Fayers PM, Haugen DF, Caraceni A, Hanks GW, Loge JH, et al. European Palliative Care Research Collaborative (EPCRC). Studies comparing numerical rating scales, verbal rating scales, and visual analogue scales for assessment of pain intensity in adults: a systematic literature review. J Pain Symptom Manage. 2011;41(6):1073-93.

20. Dos Santos Calderon P, Peixoto RF, Gomes VM, da Mota Corrêa, AS, de Alencar EN, Rossetti LM, Conti PCR. Concordance among different pain scales in patients with dental pain. J Orofac Pain. 2012;26(2):126-31.

21. Seltzer S, Bender IB, Ehrenreich J. Incidence and duration of pain following endodontic therapy. Relationship to treatment with sulfonamides and to other factors. Oral Surg Oral Med Oral Pathol. 1961;14:74-82.

22. Rosenberg PA. Clinical strategies for managing endodontic pain. Endodontic Topics. 2002;3:78-92 .

23. Siqueira Jr JF, Rôças IN, Favieri A, Machado AG, Gahyva SM, Oliveira JCM, Abad EC. Incidence of postoperative pain after intracanal procedures based on an antimicrobial strategy. J Endod. 2002;28(6):457-60.

24. Shokraneh A, Ajami M, Farhadi N, Hosseini M, Rohani B. Postoperative endodontic pain of three different instrumentation techniques in asymptomatic necrotic mandibular molars with periapical lesion: A prospective, randomized, double-blind clinical trial. Clin Oral Investig. 2017;21(1):413-8.

25. Pasqualini D, Mollo L, Scotti N, Cantatore G, Castellucci A, Migliaretti G, et al.Postoperative pain after manual and mecha nical glide path: a randomized clinical trial. $\mathrm{J}$ Endod. 2012;38(1):32-6.

26. Al-Jabreen TM. Single visit endodontics: Incidence of post-operative pain after instrumentation with three different techniques: An objective evaluation study. Saudi Dent J. 2002;14(3):136-9.

27. Kashefinejad M, Harandi A, Eram S, Bijani A. Comparison of single visit post endodontic pain using Mtwo rotary and hand K-file instruments: a randomized clinical trial. J Dent (Tehran). 2016;13(1):10-7.

28. Capar ID, Arslan H, Akcay M, Ertas H. An in vitro comparison of apically extruded debris and instrumentation times with ProTaper universal, ProTaper next, twisted file adaptive, and HyFlex instruments. $\mathrm{J}$ Endod. 
2014;40(10):1638-41.

29. Ahmed MA, Dall AQ, Khoso NA, Jouhar R. Comparison of postoperative pain after Protaper rotary and manual step-back root canal preparation techniques in single visit endodontics. J Pak Dent Assoc. 2012;21(2):103-7.

30. Talebzadeh B, Nezafati S, Rahimi S, Shahi S, Lotfi M, Ghasemi N. Comparison of manual and rotary instrumentation on postoperative pain in teeth with asymptomatic irreversible pulpitis: A randomized clinical trial. Iran Endod J. 2016;11(4):273-9.

31. Saha SG, Gupta RK, Bhardwaj A, Misuriya A, Saha MK, Nirwan AS. Comparison of the incidence of postoperative pain after using a continuous rotary system, a reciprocating system, and a Self-Adjusting File system in single-visit endodontics: A prospective randomized clinical trial. J Conserv Dent. 2018;21(3): 333-8.

32. Iqbal M, Kurtz E, Kohli M. Incidence and factors related to flare-ups in a graduate endodontic programme. Int Endod J. 2009;42(2):99-104.

33. Gotler M, Bar-Gil B, Ashkenazi M. Postoperative pain after root canal treatment: a prospective cohort study. Int J Dent. 2012;2012:310467.

34. Alí A, Olivieri JG, Duran-Sindreu F, Abella F, Roig M, García-Font M. Influence of preoperative pain intensity on postoperative pain after root canal treatment: a prospective clinical study. J Dent. 2016;45:39-42.

35. Mattscheck DJ, Law AS, Noblett WC. Retreatment versus initial root canal treatment: factors affecting posttreatment pain. Oral Surg Oral Med Oral Pathol Oral Radiol Endod. 2001;92(3):321-4.

36. Martín-González,

J, Echevarría-Pérez

M, Sánchez-Domínguez $\quad B$, TarilonteDelgado ML, Castellanos-Cosano L, López-
Frías FJ, Segura-Egea JJ. Influence of root canal instrumentation and obturation techniques on intra-operative pain during endodontic therapy. Med Oral Patol Oral Cir Bucal. 2012;17(5):e912-8.

37. Arias A, de la Macorra JC, Hidalgo JJ, Azabal M. Predictive models of pain following root canal treatment: a prospective clinical study. Int Endod J. 2013;46(8):784-93.

38. Carrotte P. Endodontics: Part 4. Morphology of the root canal system. $\mathrm{Br}$ Dent J. 2004;197(7):379-83.

39. Yesilsoy C, Koren LZ, Morse DR, Rankow H, Bolanos OR, Furst ML. Post-endodontic obturation pain: a comparative evaluation. Quintessence Int. 1988;19(6):431-8

40. Ng YL, Glennon JP, Setchell DJ, Gulabivala K. Prevalence of and factors affecting postobturation pain in patients undergoing root canal treatment. Int Endod J. 2004;37(6):38191.

41. Riaz A, Maxood A, Abdullah S, Saba K, Din SU, Zahid S. Comparison of frequency of post-obturation pain of single versus multiple visit root canal treatment of necrotic teeth with infected root canals. A Randomized Controlled Trial. J Pak Med Assoc. 2018;68(10):1429-33.

42. Wong AW, Zhang S, Li SK, Zhu X, Zhang $\mathrm{C}$, Chu $\mathrm{CH}$. Incidence of post-obturation pain after single-visit versus multiple-visit nonsurgical endodontic treatments. BMC Oral Health. 2015;15:96.

43. El Mubarak AH, Abu-bakr NH, Ibrahim YE. Postoperative Pain in Multiple-visit and Single-visit Root Canal Treatment. J Endod. 2010;36(1):36-9.

44. Su Y, Wang C, Ye L. Healing Rate and Postobturation Pain of Single- versus Multiplevisit Endodontic Treatment for Infected Root Canals: A Systematic Review. J Endod. 2011;37(2):125-32. 
45. Erdem Hepsenoglu Y, Eyuboglu TF, Özcan M. Postoperative pain intensity after singleversus 2-visit nonsurgical endodontic retreatment: a randomized clinical trial. $\mathrm{J}$ Endod. 2018;44(9):1339-46.

46. Alomaym MAA, Aldohan MFM, Alharbi MJ, Alharbi NA Single versus multiple sitting endodontic treatment: incidence of postoperative pain - a randomized controlled trial. J Int Soc Prev Community Dent. 2019;9(2):172-7.

47. Yoldas O, Topuz A, Isçi AS, Oztunc H. Postoperative pain after endodontic retreatment: single- versus two-visit treatment. Oral Surg Oral Med Oral Pathol Oral Radiol Endod. 2004;98(4):483-7.

48. Eleazer PD, Eleazer KR. Flare-up rate in pulpally necrotic molars in one-visit versus two-visit endodontic treatment. J
Endod. 1998;24(9):614-6.

49. García-Font M, Duran-Sindreu F, Calvo C, Basilio J, Abella F, Ali A, Roig M, Olivieri JG. Comparison of postoperative pain after root canal treatment using reciprocating instruments based on operator's experience: A prospective clinical study. J Clin Exp Dent. 2017 Jul 1;9(7):e869-e874.

\section{Correspondência para:}

Fernanda Geraldo Pappen

e-mail: ferpappen@yahoo.com.br

Faculdade de Odontologia

Universidade Federal de Pelotas

Rua Gonçalves Chaves 457, sala 507

96015-560 Pelotas/RS 